\title{
Fragmentation of Plasmid DNA Produced by Gamma Radiation: A Theoretical Approach
}

\author{
R. A. S. Silva, ${ }^{1}$ J. D. T. Arruda-Neto, ${ }^{1,2}$ and L. Nieto ${ }^{3}$ \\ ${ }^{1}$ Physics Institute, Rua do Matão, Travessa R, No. 187, University of São Paulo, 05508-090 São Paulo, SP, Brazil \\ ${ }^{2}$ FESP, São Paulo Engineering School, São Paulo, SP, Brazil \\ ${ }^{3}$ Laboratory of Devices Research, University of Campinas, Campinas, SP, Brazil
}

Correspondence should be addressed to R. A. S. Silva, ri_cardo77@hotmail.com

Received 16 August 2011; Accepted 18 September 2011

Academic Editor: E. Dague

Copyright ( 2012 R. A. S. Silva et al. This is an open access article distributed under the Creative Commons Attribution License, which permits unrestricted use, distribution, and reproduction in any medium, provided the original work is properly cited.

\begin{abstract}
Breaks in DNA, resulting in fragmented parts, can be produced by ionizing radiation which, in turn, is the starting point in the search for novel physical aspects of DNA strands. Double-strand breaks in particular cause disruption of the DNA strand, splitting it into several fragments. In order to study effects produced by radiation in plasmid DNA, a new simple mechanical model for this molecule is proposed. In this model, a Morse-like potential and a high-LET component are used to describe the DNA-radiation interaction. Two power laws, used to fit results of the model, suggest that, firstly, distribution of fragment size is nonextensive and, secondly, that a transition phase is present in the DNA fragment distribution pattern.
\end{abstract}

\section{Introduction}

One of the most important molecules for living beings is DNA, and as a result it has been widely studied. All genetic information controlling cellular reproduction is in DNA, and this is the most sensitive molecule in cell nuclei [1-3]. The interaction of radiation with organic molecules and the consequences of this interaction for biological systems have been thoroughly investigated.

Ionizing radiations such as gammas, neutrons, or alpha particles can produce breaks in DNA resulting in fragmented parts that would be the starting point in the search for novel physical aspects of DNA strands. Among these, nonextensive properties (nonlinearities) at nanoscale as a likely possibility would be mentioned. In fact, some evidence in this direction could be inferred from [4].

It is commonly accepted that secondary electrons formed in the process of ionization are mostly responsible for DNA damage, either by directly breaking the DNA strands, or by reacting with water molecules, producing more secondary electrons and free radicals, which, in turn, could provoke additional damage to DNA. Many different kinds of lesions in DNA are caused by ionizing radiation, more especially base damage and strand breaks. The latter are particularly important because they are irreparable when produced in large and clustered quantities [5]. According to experimental evidence, initial-level breaks and residual/unrepaired-level breaks, can be correlated with the various biological end-points such as loss of reproductivity, cell death, capacity, mutations, or carcinogenesis [6]. Nowadays, DNA-radiation interaction is, however, not totally understood. A detailed study of damage produced by radiation is very important for maintenance of life. It is necessary to stress that DNA damage depends on the kind of radiation (alpha, beta, or gamma) and also that electrons are fundamental in this damage mechanism [7].

Theoretical studies in radiation biology with high-LET charged particles have been helpful in understanding some types of DNA damage as double-strand breaks [8]. High-LET radiation features a highly structured deposition of energy along the charged particle tracks [9]. Moreover, high-LET radiation is expected to induce more severe and complex lesions correlated along individual particle tracks because of energy deposition characteristics [9-11]. A high quantity of biological damage is induced at sites close to particle trajectory, and damage clustering is caused by this energy localization. Double-strand breaks in particular cause disruption 
of the DNA strand, separating it into several fragments. Size distribution pattern of these fragments could reveal important aspects of the breaking mechanisms as discussed in this paper.

Presently, DNA fragmentation can be easily quantified through atomic force microscopy (AFM). This technique has been largely used in biological science [12], combining high spatial resolution with the ability to image biological samples in their physiological environment. Radiobiological research uses this powerful AFM imaging capability [13]. In fact, the investigation of DSBs induced by X-rays, neutrons, electrons, and alpha particles in plasmid DNA has been performed with AFM [14-16]. Results on damage induced in plasmid DNA by heavy ions have also been reported elsewhere [17].

Results for fragment size distribution functions with electrons and neutrons were obtained by Pang and collaborators [16]. As discussed in this paper, their results present leads for the occurrence of nonextensivity and crossover at nanoscale. In this regard, it is noted that there are several studies in the literature showing nonlinear aspects of DNA molecule [18]. For instance, studies of long-range correlations in DNA indicate that the length $(L)$ of homogeneous sequences in noncoding regions obeys a power-law-type distribution (a fingerprint for nonextensiveness), while in widely encoded regions these distributions are exponential [4].

Thus, here, a simple mechanic model for DNA is proposed, aiming at the understanding of DNA physical characteristics when exposed to different radiation intensities (doses), particularly aspects associated with its fragmentation pattern, for example, the occurrence, or not, of nonextensivity at molecular level. The model simulates DNA strands by spring-mass lines. These masses, representing nucleotides, are connected by two components of elastic potential-one is the conventional linear harmonic oscillator and the other the nonlinear Morse potential. The former holds masses together, and the latter takes into account the interaction between masses and radiation particles. The reason for choosing a Morse potential is because of its ability to simulate stretching and compression of covalent bonds $[19,20]$.

The results of our model calculations are compared with those from experiments carried out with gammas in our laboratory, and with electrons and neutrons from the literature [21].

\section{The Model}

In the present model, each DNA base pair is represented by a mass $m$. These masses are joined together along a strand by spring potentials with coupling constants $k$. Furthermore, there is a nonlinear potential relating the distance between masses and charged particles. Base pair displacements are only longitudinal; that is, they would be along the helix axis. Each mass $\left(m_{i}\right)$ has a linear displacement, denoted by $x$, while $r_{i}$ is the approximate distance of the radiation particles to $m_{i}$.

We propose the DNA-radiation interaction leading to the breaking of covalent bonds be given by a Morse-like potential $V\left(x_{i}, r_{i}\right)$. This potential is generally chosen to simulate covalent $[19,20]$ bonds and hydrogen bonds in small molecules [22, 23].

For our purposes, it is assumed that the potential depends on a combination of variables $x_{i}$ and $r_{i}$, that is, $V\left(x_{i}+g\left(r_{i}\right)\right)$ thus, the Morse-like potential is given by

$$
V\left(x_{i}+\mathrm{g}\left(r_{i}\right)\right)=D_{M}\left(e^{-a\left(x_{i}+g\left(r_{i}\right)\right)}-1\right)^{2},
$$

where $D_{M}$ is the dissociation energy of a base pair in a given strand and $a$ is a parameter with dimension of inverted length. $g(r)$, a function defined by

$$
g(r)=\frac{K}{r}
$$

is introduced to consider interactions between charged particle approximation and DNA. In this case, $K=D$. LET - $C$, where $D$ is related to radiation dose, LET is the average linear energy transfer [24], and $C=(3.43 / 7.504)$ $\AA^{3} \mathrm{eV}^{-1}(3.4 \AA$ is the average distance between the adjacent bases in the same DNA strand, and $7.504 \mathrm{eV}$ is the necessary energy to break a covalent bond [25].

The Hamiltonian for the system described above can be written as

$$
H=H_{p}+H_{v}
$$

where

$$
H_{p}=\sum_{i}\left\{\frac{p_{x_{i}}^{2}}{2 m}\right\}
$$

represents the kinetic energy of the system, and

$$
H_{V}=\sum_{i}\left\{\frac{k}{2}\left[x_{i}-x_{i-1}\right]^{2}+V\left(x_{i}, r_{i}\right)\right\}
$$

is the potential energy. In (2) $p_{x}$ is the linear momentum. For the sake of simplicity, the system is considered homogenous, meaning that masses $m$ and strength constants $k$ are the same for all nucleotides. The ionizing radiations (alpha, beta, and gamma) can be defined by a LET component [24] in the Morse potential.

For a chain with $N$ base pairs, the classical partition function is given in terms of the Hamiltonian introduced in (1) and may be factored as

$$
Z=\int_{-\infty}^{\infty} \coprod_{n=1}^{N} d x_{n} d p_{n} e^{-H / k_{B} T}=Z_{x} Z_{p}
$$

These integrals for all momenta are merely Gaussian integrals, providing

$$
Z_{p}=\left(2 \pi m k_{B} T\right)^{N / 2},
$$

where $k_{B}$ is the Boltzmann constant. The term in the $x$ variables, $Z_{x}$, comes from a harmonic chain of oscillators with an on-site nonlinear potential for each oscillator. Therefore, partition function $Z_{x}$ is written as

$$
Z_{x}=\int \coprod_{n=1}^{N} d x_{n} e^{-f\left(x_{i}, x_{i-1}, r_{i}\right) / k_{B} T},
$$


where $f$ is given by

$$
f\left(x_{i}, x_{i-1}, r_{i}\right)=\frac{k}{2}\left(x_{i}-x_{i-1}\right)^{2}+V\left(x_{i}, r_{i}\right)
$$

Integrals in $Z_{x}$ can be calculated using eigenfunctions and eigenvalues of a transfer integral operator $[26,27]$ :

$$
\int d_{x-1} e^{-f\left(x_{i}, x_{i-1}, r_{i}\right) / k_{B} T} \psi_{i}\left(x_{i-1}\right)=e^{-\varepsilon_{i} / k_{B} T} \psi_{i}\left(x_{i}\right) .
$$

In the thermodynamic limit, $N \rightarrow \infty$, the partition function $Z_{x}$ is reduced to

$$
Z_{x}=e^{-N \varepsilon_{0} / k_{B} T}
$$

where $\varepsilon_{0}$ is the ground-state eigenvalue for a Schrödinger-like equation given by

$$
\left\{-\frac{\left(k_{B} T\right)^{2}}{2 k} \frac{d^{2}}{d x_{i}^{2}}+V\left(x_{i}, g\left(r_{i}\right)\right)\right\} \psi_{0}\left(x_{i}\right)=\left(\varepsilon_{0}-\sigma_{0}\right) \psi_{0}\left(x_{i}\right)
$$

with

$$
\sigma_{0}=\frac{1}{2} k_{B} T \ln \left(\frac{k}{2 \pi k_{B} T}\right) .
$$

The eigenvalue and the normalized eigenfunction for the ground state are, respectively,

$$
\begin{gathered}
\mathcal{E}_{0}=\sigma_{0}+k_{B} T a \sqrt{\frac{D}{2 k}}-\frac{\left(k_{B} T a\right)^{2}}{8 k}, \\
\psi_{0}\left(x_{i}\right)=C e^{\left[-\delta e^{-a(x+g(r))}-a x(\delta-0.5)\right]}
\end{gathered}
$$

with $\delta=(2 k D)^{1 / 2} /\left(k_{B} T a\right)$ and $C$ the normalization constant. In the limit $N \rightarrow \infty$, the average stretching $\langle x\rangle$ will be dependent only on the ground-state eigenfunction as

$$
\langle x\rangle=\int_{-\infty}^{\infty} \psi_{0}^{2}(x) x d x
$$

It is suggested that the mean $\langle x\rangle$ could be related to the number of fragments produced by radiation. In the absence of any external interference, the average distance between neighboring nucleotides in the same DNA strand is $3.4 \AA$, and for a linearized DNA with $L=1000 \mathrm{~nm}$, the number $N$ of links involving the neighboring nucleotides is given by $N=1000 \mathrm{~nm} /(3.4 \AA)=2941$. It is considered that the breaking probability of DNA is given by $\langle x\rangle / r$. Therefore, we can relate this rate to the value of $N$ and $L$ given above to obtain the distribution of the number of fragments and their size according to radiation dose. In this case, the number of fragments and length distribution are given, respectively, by

$$
N=N \frac{\langle x\rangle}{r}, \quad L=\frac{L}{\langle x\rangle / r} .
$$

\section{Results and Discussion}

The best values for the parameters obtained from experimental results for DNA presented in the literature as $a=$ $2.81 \AA$ and $k=0.06 \mathrm{eV} \AA^{-2}$ [28]. Covalent bonds are broken in radiation-DNA interaction, and energy values for these breaks are already known. For example, the energy to break a double covalent bond between oxygen and carbon atoms is around $724 \mathrm{~kJ} / \mathrm{mol}$ [brad](or $7504 \mathrm{eV} / \mathrm{mol}$ ). Therefore, for a preliminary theoretical investigation, it is assumed that $D_{M}=7504 \mathrm{eV}$. The value of LET used in this work is $19.6 \mathrm{keV} / \mu \mathrm{m}$ for gamma radiation [24]. The qualitative behavior of the average stretching $\langle x\rangle$ as function of the approximation distance $r$ is obtained from (16) and shown in Figure 1.

It is observed that (1) the low value for $D$ (continuous curve in Figure 1) corresponds to the lowest damage level of gamma radiation. The average values for $x(\langle x\rangle)$ are very low suggesting that only a few breaks were produced along the DNA chain; (2) the curve drawn with + symbols corresponds to a higher dose relative to the previous continuous curve, and a faster increase of $\langle x\rangle$ is observed, indicating occurrence of a higher damage level; (3) in the third case (squared symbol curve), factor $D$ was considerably increased resulting in a steeper increase of $\langle x\rangle$, which could be associated with longer exposure to radiation. Therefore, large values $\langle x\rangle$ could indicate several breaks in the DNA sequence.

The role played by radiation dose in DNA strand breakage may also be seen in Figure 2 which shows the dependence of $\langle x\rangle$ on radiation dose. In the case of high doses of radiation, with short interaction distance $(r=5 \AA)$, the values of $\langle x\rangle$ tend to be larger than the long distances $(r=$ $10 \AA$ ), indicating a higher frequency of DNA strand breakage. Moreover, only interactions of short distance ( $r$ much less than $5 \AA$ ) produce high values of $\langle x\rangle$ at low dose.

Figure 3 shows the distribution of DNA fragments as a function of radiation dose. The changes are clearly visible since the larger amount of smaller fragments is produced with longer exposure to radiation, and an increase in the number of small fragments with increasing radiation dose is observed. What is more, the results in Figure 3 show a behavior qualitatively like that of a system with damped oscillations, characteristic of a fully homogeneous system.

Very similar behavior observed for the results shown in Figure 4 was obtained by Pang and collaborators for distribution functions of fragment size using neutrons and electrons [21]. The curves shown were obtained by a simple fitting of power laws where it was found that the exponents are fractional. Two functions of power law type to describe the data were necessary, strongly suggesting the occurrence of a crossover in nanoscale. Two functions used to fit the data in Figure 3(a), and their values are shown in Table 1.

\section{Final Remarks and Conclusions}

The development of biophysical models requires a simplified description of structural properties of the elements involved, as carried out in the model developed in this paper. With the help of statistical mechanics tools, the model used here to 


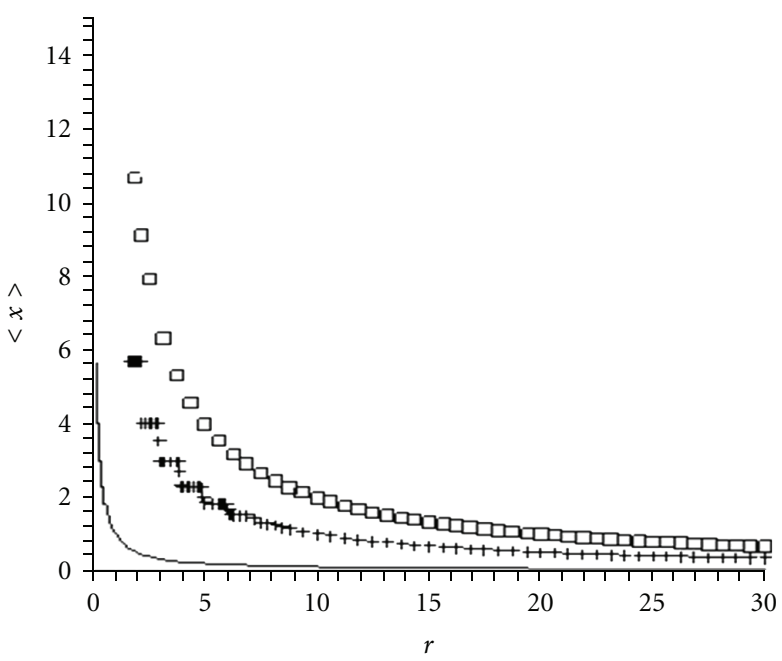

Figure 1: Average stretching between neighboring bases pairs as function of $r$ for three radiation dose: low (continuous curve), moderate $(+)$ and high (square).

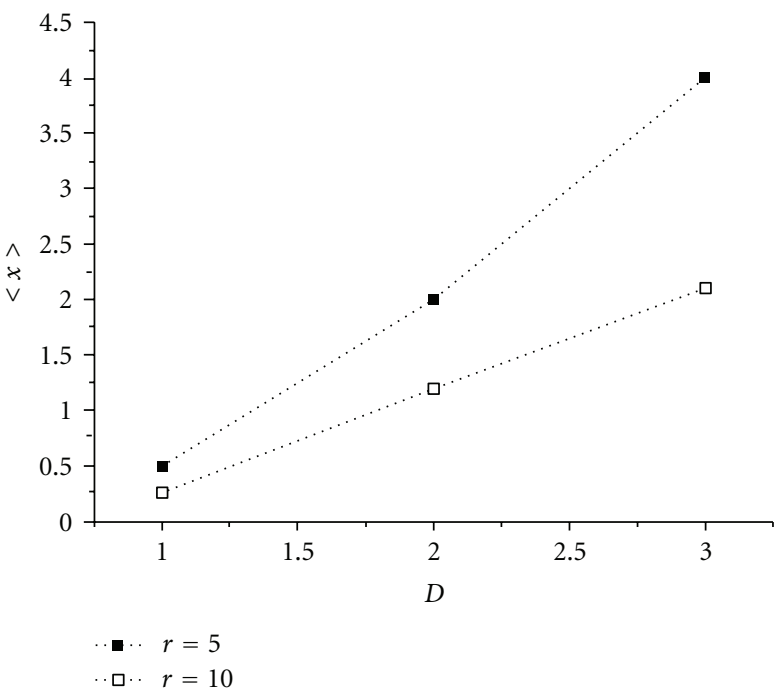

FIgURE 2: Variation of the average stretching as function of radiation dose, for $r=5$ and $r=10 \AA$.

simulate the DNA-radiation interaction has yielded results qualitatively consistent with those obtained experimentally: the higher number of smaller fragments is produced with the longer exposure to radiation, that is, with higher radiation doses.

The DNA strand behaved as a set of coupled oscillators subject to a purely harmonic interaction between each oscillator and its first neighbors. The average stretch $\langle x\rangle$ between the base pairs would be the elongation of oscillation and determines the greater or lesser propensity of the system to break.

The shape of the curve representing the number of fragment size as a function of radiation dose, as shown by the model developed here (Figure 3), is characteristic of a system

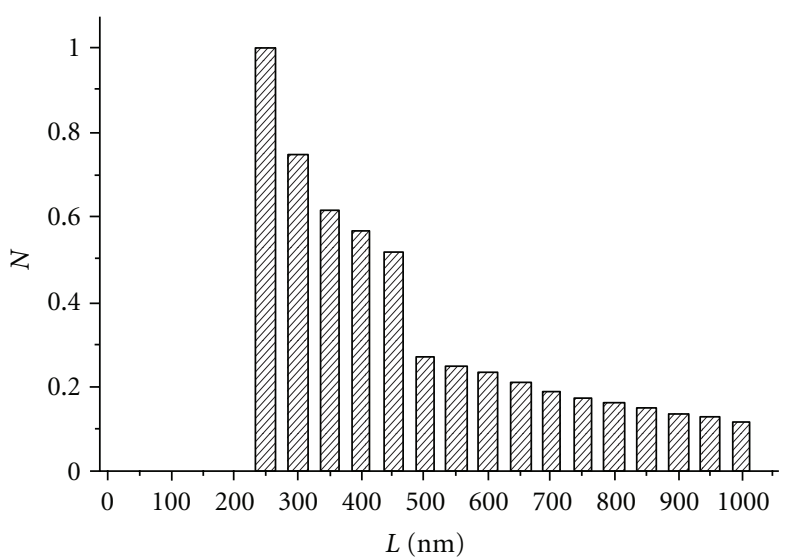

(a)

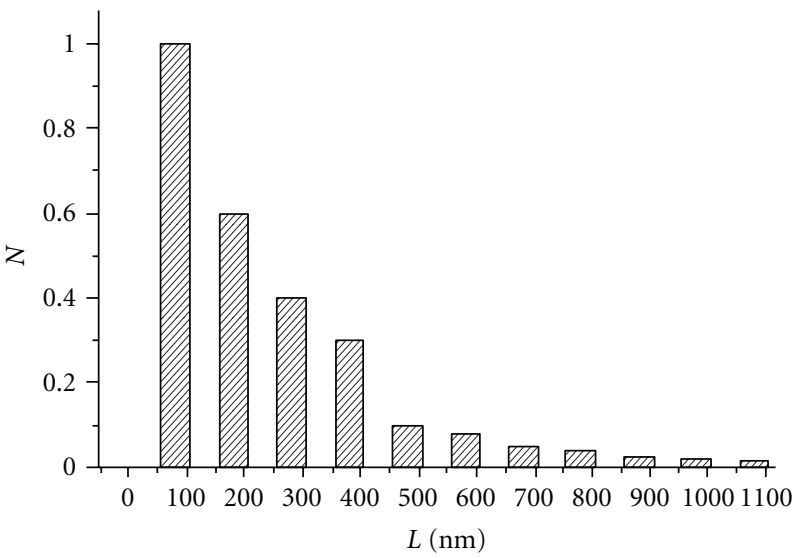

(b)

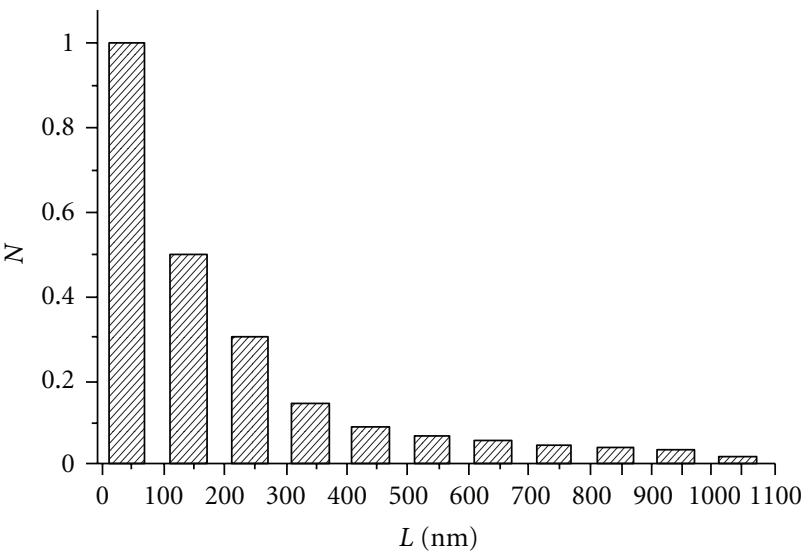

(c)

Figure 3: Number of fragments versus their length. Graphs (a), (b), and (c) refer to the low, medium, and high dose, respectively.

with damping. Two power laws used to fit results of the model suggest a nonextensive distribution of fragment size, this being a precondition for fractalization. Furthermore, obtaining two power laws for the same dose strongly suggests a transition phase in the DNA fragment distribution pattern.

It is pertinent to stress that the model does not take into account the inhomogeneity of the DNA molecule, and this property can affect the process of fragmentation; that is, 


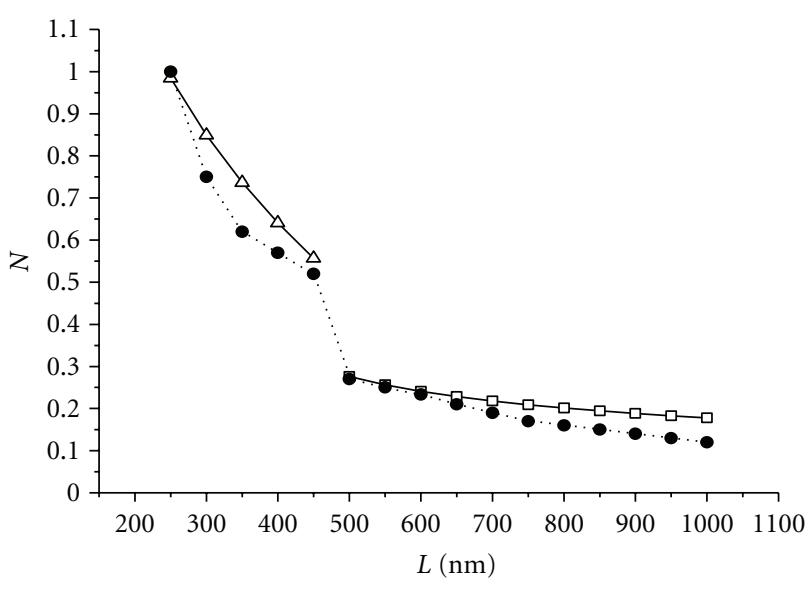

FIGURE 4: Size distribution of DNA fragments. Full circles refer to the histogram (a) of Figure 3; triangles and squares were calculated from the expressions $p(L)=c+d L^{-\alpha_{1}}$ and $p(L)=(a+b L)^{-\alpha_{2}}$, respectively. The lines (dotted and continuous) are to guide the eyes only.

TABle 1: Fit of data in Figure 3(a), using two power laws. Parameter values are, $a=-25, b=0.1, c=-6.5, d=13, \alpha_{1}=0.4$ and $\alpha_{2}=$ 0.1 .

\begin{tabular}{lcc}
\hline$L$ & $(a+b L)^{-\alpha_{1}}$ & $c+d L^{-\alpha_{2}}$ \\
\hline 1000 & 0,17782 & \\
950 & 0,18279 & \\
900 & 0,18829 & \\
850 & 0,19442 & \\
800 & 0,20131 & \\
750 & 0,20913 & \\
700 & 0,21813 & \\
650 & 0,22865 & \\
600 & 0,2412 & \\
550 & 0,25654 & 0,55703 \\
500 & 0,27595 & 0,64064 \\
450 & & 0,73663 \\
400 & & 0,84905 \\
350 & & 0,98427 \\
300 & & \\
250 & & \\
\hline
\end{tabular}

regions rich in AT (base adenine attached to base thymine) could break more easily than GC-rich regions (base guanine connected to base cytosine) since the former are linked by two hydrogen bonds while GC have three hydrogen bonds. These aspects are to be considered in a forthcoming study.

\section{Acknowledgment}

This work was supported by FAPESP and CNPq (Brazilian agencies).

\section{References}

[1] S. H. Strogatz, "Quoted by S. Nadis in "News feature: all together now"," Nature, vol. 421, pp. 780-782, 2003.

[2] C. Tsallis, "Possible generalization of Boltzmann-Gibbs statistics," Journal of Statistical Physics, vol. 52, no. 1-2, pp. 479-487, 1988.

[3] C. Tsallis, S. V. F. Levy, A. M. C. Souza, and R. Maynard, "Statistical-mechanical foundation of the ubiquity of levvy distributions in nature," Physical Review Letters, vol. 75, no. 20, pp. 3589-3593, 1995.

[4] P. Bernaola-Galván, R. Román-Roldán, and J. L. Oliver, “Compositional segmentation and long-range fractal correlations in DNA sequences," Physical Review E, vol. 53, no. 5, pp. 51815189, 1996.

[5] U. Amaldi and G. Kraft, "Radiotherapy with beams of carbon ions," Reports on Progress in Physics, vol. 68, no. 8, pp. 18611882, 2005.

[6] E. L. Alpen, Radiation Biophysics, Academic Press, San Diego, Calif, USA, 1998.

[7] Z. G. Yu and X. Song, "Variable range hopping and electrical conductivity along the DNA double helix," Physical Review Letters, vol. 86, no. 26 I, pp. 6018-6021, 2001.

[8] A. Chatterjee, "Radiobiological effects of high-let particles: DNA strand breaks," Nuclear Instruments and Methods in Physics Research A, vol. 280, no. 2-3, pp. 439-448, 1989.

[9] M. Krämer and G. Kraft, "Track structure and DNA damage," Advances in Space Research, vol. 14, no. 10, pp. 151-159, 1994.

[10] D. T. Goodhead, "The initial physical damage produced by ionizing radiations," International Journal of Radiation Biology, vol. 56, no. 5, pp. 623-634, 1989.

[11] H. Nikjoo, P. O'Neill, M. Terrissol, and D. T. Goodhead, "Quantitative modelling of DNA damage using Monte Carlo track structure method," Radiation and Environmental Biophysics, vol. 38, no. 1, pp. 31-38, 1999.

[12] H. G. Hansma and J. H. Hoh, "Biomolecular imaging with the atomic force microscope," Annual Review of Biophysics and Biomolecular Structure, vol. 23, pp. 115-139, 1994.

[13] D. Pang et al., "Atomic force microscopy imaging of DNA and DNA repair proteins: applications in radiobiological research," Radiation Oncology Investigations, vol. 5, pp. 163-169, 1997.

[14] S. Boichot, M. Fromm, S. Cunniffe et al., "Investigation of radiation damage in DNA by using atomic force microscopy," Radiation Protection Dosimetry, vol. 99, no. 1-4, pp. 143-145, 2002.

[15] D. Pang, B. L. Berman, S. Chasovskikh, J. E. Rodgers, and A. Dritschilo, "Investigation of neutron-induced damage in DNA by atomic force microscopy: experimental evidence of clustered DNA lesions," Radiation Research, vol. 150, no. 6, pp. 612-618, 1998.

[16] D. Pang, J. E. Rodgers, B. L. Berman, S. Chasovskikh, and A. Dritschilo, "Spatial distribution of radiation-induced doublestrand breaks in plasmid DNA as resolved by atomic force microscopy," Radiation Research, vol. 164, no. 6, pp. 755-765, 2005.

[17] K. Psonka, S. Brons, M. Heiss, E. Gudowska-Nowak, and G. Taucher-Scholz, "Induction of DNA damage by heavy ions measured by atomic force microscopy," Journal of Physics Condensed Matter, vol. 17, no. 18, pp. S1443-S1446, 2005.

[18] M. Gerard and A. R. Bishop, "Statistical mechanics of a nonlinear model for DNA denaturation," Physical Review Letters, vol. 62, no. 23, pp. 2755-2758, 1989. 
[19] T. C. Lim, "The relationship between Lennard-Jones (126) and Morse potencial function," Journal of Mathematical Chemistry, vol. 33, p. 1, 2003.

[20] J. A. Greathouse, J. S. Durkin, J. P. Larentzos, and R. T. Cygan, "Implementation of a Morse potential to model hydroxyl behavior in phyllosilicates," Journal of Chemical Physics, vol. 130, no. 13, Article ID 134713, 2009.

[21] D. Pang et al., "Investigation of neutron-induced damage in DNA by atomic force microscopy: experimental evidence of clustered DNA lesions," Journal of The Radiation Research Society, vol. 150, no. 6, pp. 612-618, 1998.

[22] Y. Gao, K. V. Devi-Prasad, and E. W. Prohofsky, "A selfconsistent microscopic theory of hydrogen bond melting with application to poly (dG)·poly(dC)," The Journal of Chemical Physics, vol. 80, no. 12, pp. 6291-6298, 1983.

[23] S. Zdravkovic and M. V. Sataric, "Single-molecule unzippering experiments on DNA and Peyrard-Bishop-Dauxois model," Physical Review E, vol. 73, Article ID 021905, pp. 1-11, 2006.

[24] E. L. Alpen, Radiation Biophysics, Prentice Hall, NJ, USA, 1990.

[25] J. E. Brady and G. E. Humiston, Química Geral. 2, LTC, Rio de Janeiro, Brazil, 1986.

[26] D. J. Scalapino, M. Sears, and R. A. Ferrell, "Statistical mechanics of one-dimensional ginzburg-landau fields," Physical Review B, vol. 6, no. 9, pp. 3409-3416, 1972.

[27] M. Peyrard, "Nonlinear dynamics and statistical physics of DNA," Nonlinearity, vol. 17, no. 2, pp. R1-R40, 2004.

[28] R. A. S. Silva, E. D. Filho, and J. R. Ruggiero, "A model coupling vibrational and rotational motion for the DNA molecule," Journal of Biological Physics, vol. 34, no. 5, pp. 511-519, 2008. 

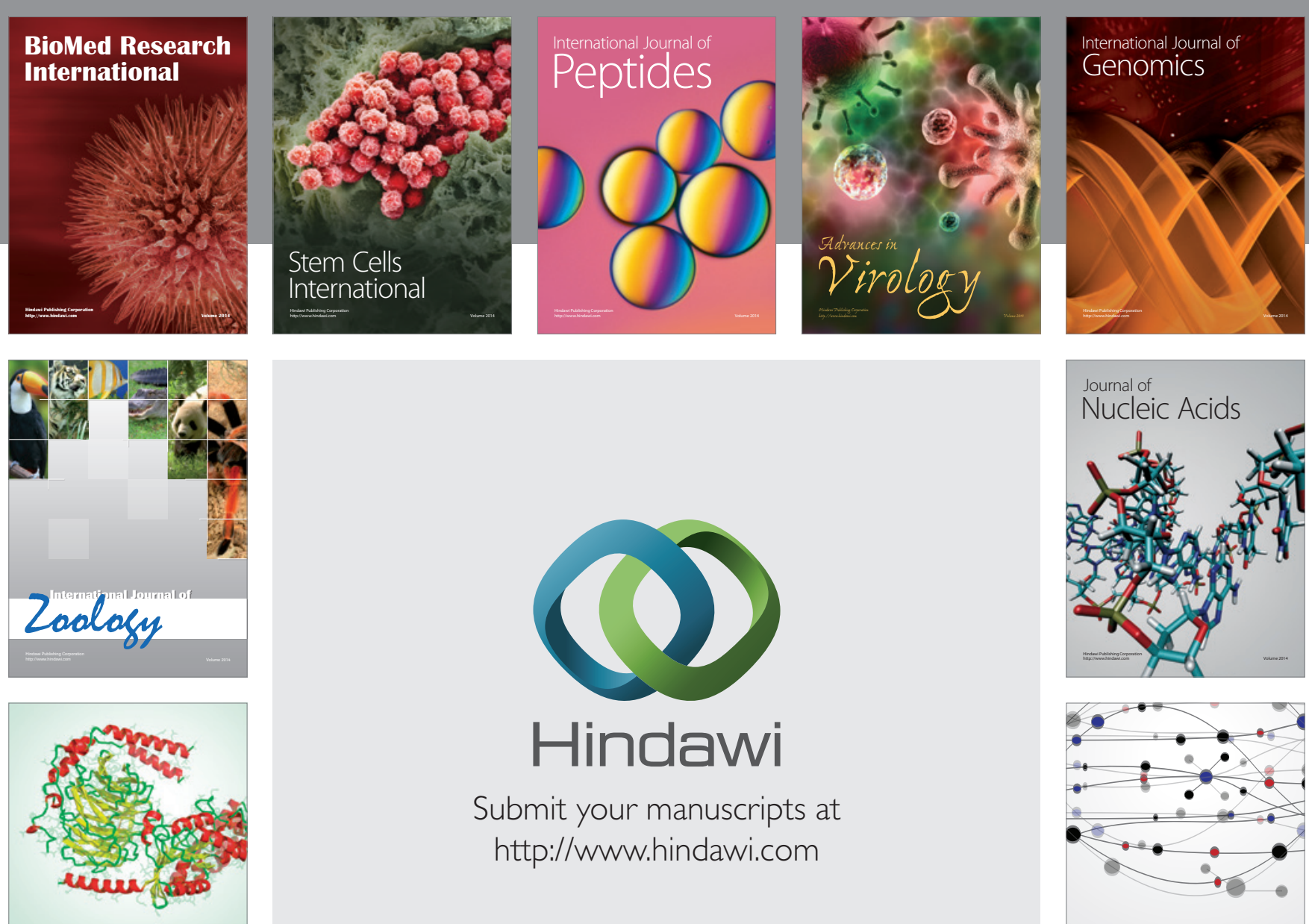

Submit your manuscripts at

http://www.hindawi.com

Signal ${ }^{\text {Jumal }}$ Transduction
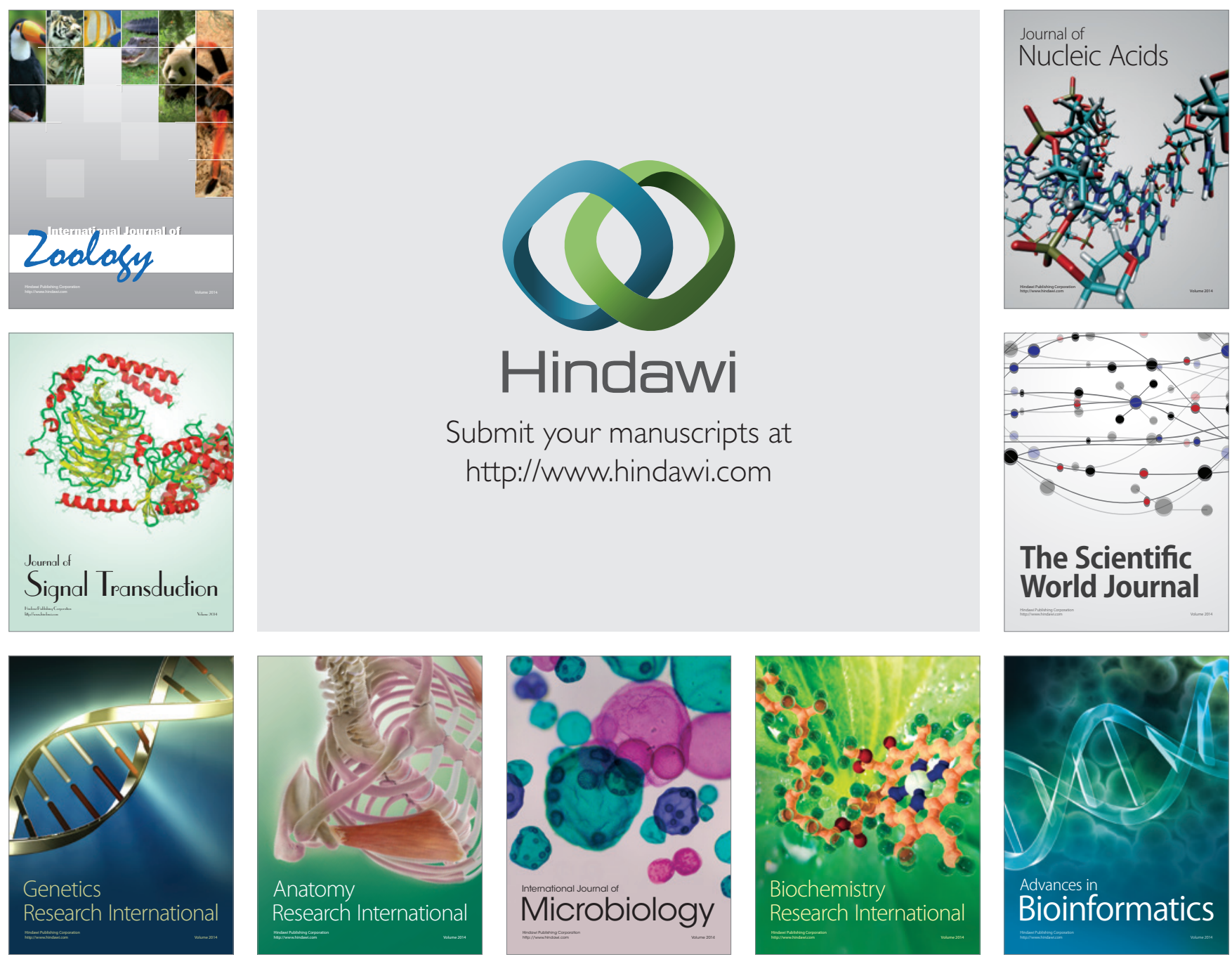

The Scientific World Journal
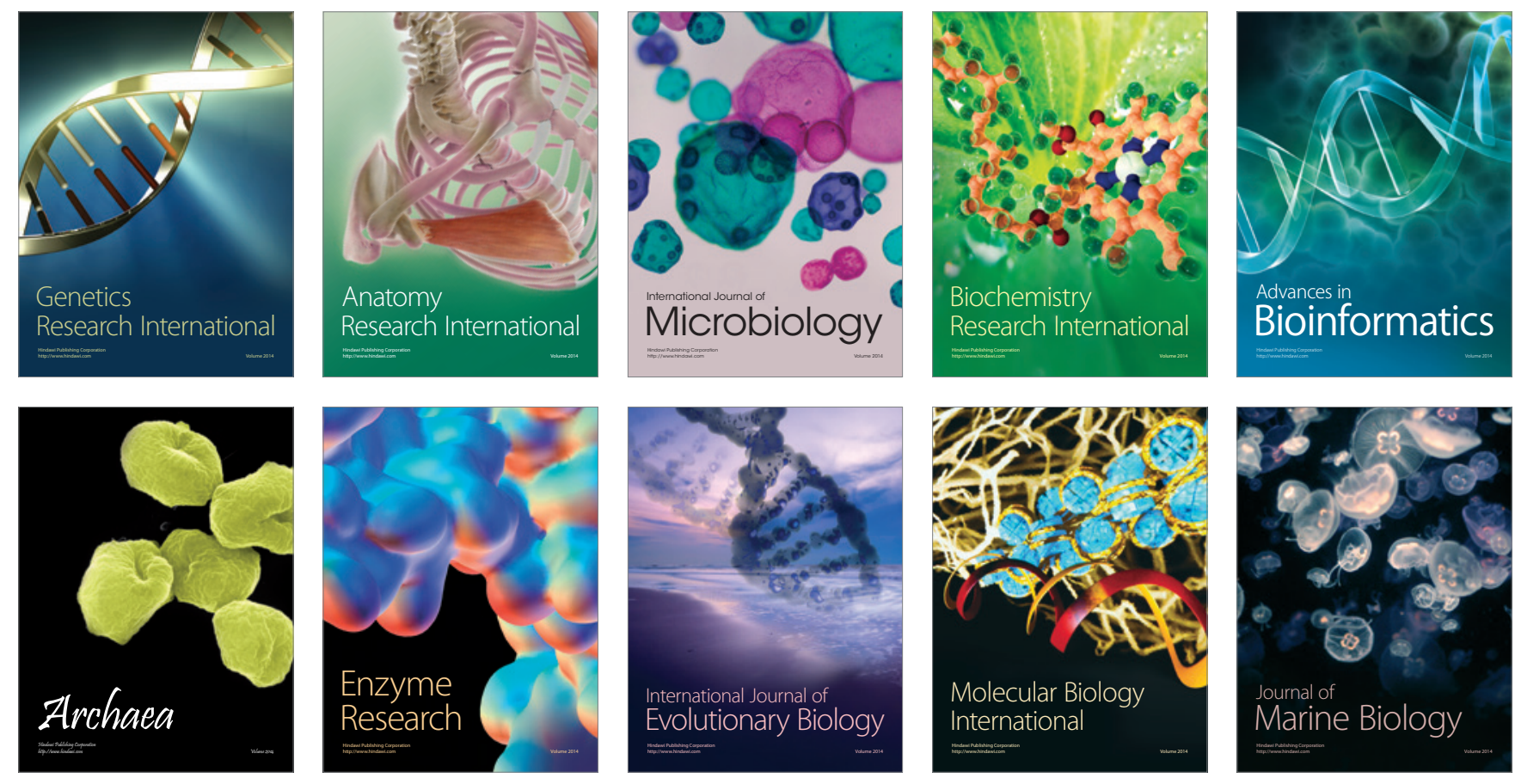